\title{
Prevalência de consumo de bebidas alcoólicas e de alcoolismo em uma região metropolitana do Brasil *
}

\section{Prevalence of the consumption of alcoholic beverages and of alcoholism in an urban region of Brazil}

\author{
Liz Maria de Almeida**, Evandro da S. F. Coutinho***
}

\begin{abstract}
ALMEIDA, L. M. de \& COUTINHO, E. da S. F. Prevalência de consumo de bebidas alcoólicas e de alcoolismo em uma região metropolitana do Brasil. Rev. Saúde Pública, 27: 23-9, 1993. Foi realizado Inquérito epidemiológico com o objetivo de estimar o uso de substâncias psicoativas e a prevalência de alcoolismo. Tomou-se como referência a população de maiores de 13 anos de idade de região administrativa da cidade do Rio de Janeiro, RJ (Brasil), da qual se extraiu uma amostra aleatória de 1.459 indivíduos. Os resultados referentes ao uso de bebidas alcoólicas e alcoolismo, identificados através do teste CAGE, mostraram: prevalência de 51\% para o consumo de álcool e de $3 \%$ para alcoolismo, sendo $4,9 \%$ em homens e $1,7 \%$ em mulheres; maior proporção de consumidores de álcool e de alcoolistas entre homens de 30 e 49 anos; abstinência mais freqüente entre os viúvos, protestantes e indivíduos com níveis de renda inferiores.
\end{abstract}

Descritores: Alcoolismo, epidemiologia. Consumo de bebidas alcólicas, epidemiologia.

\section{Introduçăo}

A epidemiologia tem sido importante na caracterização do abuso e dependência de bebidas alcoólicas como um problema de saúde pública. As análises de dados secundários apontam o alcoolismo como a segunda causa de internação psiquiátrica, como uma das principais causas de aposentadoria por invalidez ${ }^{5}$, do absenteísmo, dos acidentes de trabalho e de trânsito ${ }^{17}$.

Os coeficientes de prevalência de alcoolismo diferem entre os países. A Organização Panamericana da Saúde (citada por Santana ${ }^{22}$ ) divulgou dados em que se verifica uma variação de 1 a $10 \%$ da população total caracterizada como alcoolistas ou "grandes bebedores".

Schuckit ${ }^{25}$ afirma que, com um critério rigoroso, 5 a $10 \%$ da população masculina adulta dos Estados Unidos apresentam sintomas de alcoolismo em al-

\footnotetext{
* Pesquisa financiada pela Fundação Kellogg.

* Núcleo de Estudos de Saúde Coletiva da Universidade Federal do Rio de Janeiro - Rio de Janeiro, RJ - Brasil.

*** Departamento de Epidemiologia e Métodos Quantitativos em Saúde da Escola Nacional de Saúde Pública - FIOCRUZ e do Instituto de Medicina Social da Universidade do Estado do Rio de Janeiro - Rio de Janeiro, RJ.
}

Separatas/Reprints: L. M. do Almeida - Rua de Paranhos Antunes, 131 - Ap. 201 - Barra da Tijuca 22620-300 - Rio de Janeiro, RJ - Brasil. gum momento de sua vida. Os coeficientes mais elevados são encontrados em homens, de 30 a 50 anos, católicos, com menor nível de instrução e de renda.

O National Hearth Foundation of Australia e o Australian Institute of Health ${ }^{19}$ realizaram, em 1989, um inquérito sobre prevalência de fatores de risco para doenças cardiovasculares, onde apenas $1 \%$ dos homens e menos ainda das mulheres era bebedores de alto risco (numa relação entre frequiência e quantidade de bebidas ingeridas).

$\mathrm{Na}$ América Latina encontramos dados de inquéritos epidemiológicos mais recentes. $\mathrm{Na}$ Costa Rica o Instituto Nacional sobre Alcoolismo estudou os coeficientes de prevalência de três cidades. ${ }^{18} \mathrm{Na}$ população de Alajuela ${ }^{9}$, acima de 15 anos, a prevalência de alcoolismo crônico foi de $4.6 \%$, sendo mais frequiente entre os indivíduos do sexo masculino, de 30 a 44 anos, sem religião, casados, com baixo nível de instrução e trabalhando em nível secundário. Em Quespos ${ }^{11}$ o citado Instituto encontrou $11 \%$ de alcoolistas crônicos, mais freqüentes entre homens com idade entre 45 e 59 anos, de religião católica e separados. Numa terceira cidade-Canton de Parrita ${ }^{10}$ a prevalência foi de $21,2 \%$, com predominância em indivíduos do sexo masculino, na faixa etária de 30 a 44 anos, católicos, com nível de instrução mais elevado, casados e com maior tempo de residência.

Galvis e Murrelle ${ }^{8}$ estudaram a população urbana da Colômbia entre 12 e 64 anos, em 1987. O instrumento utilizado foi o teste CAGE, com pon- 
to de corte entre 1 e 2 pontos para caracterizar "alto risco" e entre 2 e 3 pontos como critério para o diagnóstico de alcoolismo. Esses autores estimaram em $7,3 \%$ a prevalência de bebedores de "alto risco" e $8 \%$ de "alcoolistas".

No Equador, Aguilar ${ }^{1}$ investigou uma amostra representativa da população entre 10 e 65 anos, e encontrou $13 \%$ de dependência ao álcool. No México, um inquérito nacional na população entre 18 e 65 anos ${ }^{16}$ identificou $11 \%$ de homens e $0,6 \%$ de mulheres alcoolistas, segundo os critérios da IX Classificação Internacional de Doenças (CID-9).

No Brasil os estudos com amostras da população geral são escassos. Além do mais, a diversidade geográfica, socioeconômica e cultural não nos permite generalizações. A maioria dos estudos tem encontrado prevalências para o alcoolismo entre 3 e $6 \%{ }^{22}$. Todos os inquéritos realizados apontaram diferenças para a variável sexo, mas os achados para as variáveis socioeconômicas não têm se mostrado consistentes.

Azoubel Neto $^{3}$ encontrou, em Ribeirão Preto, $6,2 \%$ de alcoolistas crônicos. Luz Jr..$^{13}$ observou a mesma prevalência, sendo $11 \%$ para homens e $1,5 \%$ para mulheres. Numa área de prostituição de Salvador, Coutinho (citado por Santana ${ }^{22}$ ) estimou em $22,6 \%$ a prevalência do alcoolismo, sendo $27,7 \%$ para o sexo masculino e $20,1 \%$ para ofeminino. Também em Salvador, Santana ${ }^{24}$ obteve uma proporção de 3,4\% de alcoolistas na população acima de 14 anos em um bairro de baixa renda, sendo $6 \%$ para os homens e $0,7 \%$ para as mulheres. Em outro estudo, também em Sal vador, Santana e col..$^{23}$ encontraram uma prevalência de $6,2 \%$ que, estratificada por sexo, foi de $11 \%$ e $1,5 \%$ para o sexo masculino e feminino, respectivamente.

Com o objetivo de estimar a prevalência do consumo de álcool e do alcoolismo numa população geral, assim como traçar um perfil desses usuários, realizou-se um estudo seccional (inquérito epidemiológico). No presente trabalho estão apresentados os dados desse estudo na região do Município do Rio de Janeiro, Ilha do Governador.

\section{Materlal e Método}

\section{Amostra}

População de referência. A população investigada era composta de moradores da Ilha do Governador, na cidade do Rio de Janeiro-Brasil, com mais de 13 anos de idade. No presente trabalho, foi estudada uma amostra de cerca de 250.000 habitantes. A Ilha do Governador tem uma população bastante heterogênea quanto ao nível socioeconômico, distribuída por 19 bairros e 13 favelas.
Delineamento. Utilizou-se um delineamento amostral em três estágios. No primeiro sorteou-se 59 setores censitários de um total de 183 , sorteio esse com probabilidade proporcional ao tamanho de cada conglomerado, o qual foi estimado de acordo com dados fornecidos aos autores pela Fundação IBGE. Foram excluídos os setores coletivos como hospitais, escolas, quartéis, igreja. Em seguida sortearam-se 30 domicílios em cada setor, selecionando-se um individuo aleatoriamente dentro de cada domicílio.

Tamanho. Para cálculo do tamanho amostral foram considerados os seguintes parâmetros:

- prevalência em homens $=6 \%$

- prevalência em mulheres $=4 \%$

- erro amostral $=2 \%$

- efeito de delineamento $=2$

Assim, obteve-se um tamanho amostral de 1.800 indivíduos com mais de 13 anos.

\section{Instrumento}

Para avaliar o consumo de substâncias psicoativas e a prevalência de alcoolismo aplicou-se a cada indivíduo sorteado um questionário com 52 perguntas fechadas que incluíam, entre outras, questões sobre dados socioeconômicos e o teste CAGE. O consumo aqui abordado refere-se somente ao fato do entrevistado ter declarado fazer uso de bebida alcoólica, isto é, não ser abstêmio. Não se avaliou a quantidade, a frequiência ou a duração do consumo. $O$ teste CAGE foi utilizado como critério para categorizar os entrevistados como alcoolistas ou não. Trata-se de questionário, composto por quatro perguntas*, desenvolvido em 1968, que considera como caso suspeito de alcoolismo alguém que responda afirmativamente a duas ou mais perguntas. No Brasil sua validação foi feita por Masur e Monteiro $^{15}$, que encontraram uma sensibilidade de $88 \%$ e uma especificidade de $83 \%$. Estudos de validação em outros países têm obtido valores semelhantes para a sensibilidade, e uma especificidade entre 90 e $95 \% 4,12,14,21$. Entretanto, tais es-

* 1. Alguma vez o(a) senhor(a) sentiu que deveria diminuir a quantidade de bebida alcólica ou parar de beber?

2. As pessoas $o(a)$ aborrecem porque criticam o seu modo de tomar bebidas alcoólicas?

3. $O(a)$ senhor(a) se sente chateado(a) consigo mesmo(a) pela maneira como costuma tomar bebidas alcoólicas?

4. Costuma tomar bebidas alcoólicas pela manhã para diminuir o nervosismo ou ressaca? 
tudos não têm avaliado o seu desempenho em populações gerais. No caso da presente investigação, o estudo de validação do teste CAGE não foi realizado por insuficiência de recursos financeiros e dificuldades operacionais.

\section{Coleta de dados}

Dezoito estudantes da área biomédica da Universidade Federal do Rio de Janeiro foram treinados para mapear os setores censitários sorteados e aplicar o questionário. A atualização dos mapas durou três meses e a coleta dos dados, incluindo até três visitas a cada domicílio, quatro meses. O trabalho de campo foi supervisionado por três profissionais, sendo um psiquiatra e dois psicólogos.

\section{Análise dos dados}

Os dados coletados foram organizados em microcomputador. Em seguida foram feitas tabulaçōes de freqüências simples e tabulações cruzadas.

A associação do consumo de bebida alcoólica e do alcoolismo com as variáveis demográficas e socioeconômicas foi estudada através dos "oddsratio" ou razão de produtos cruzados (RPC). Essa medida, embora utilizada com maior freqüência em estudos de delineamento caso-controle, pode ser adotada para estimar a associação entre variáveis categóricas, além de possibilitar o uso de regressão logística. $O$ grau de significância das RPC não ajustadas foi avaliado pelo teste do qui-quadrado.

$O$ efeito de fatores potencialmente "confounding" e de interações foi estudado, inicialmente, através de análise estratificada com estimação de "odds-ratio" de Mantel-Haenszel. Para isso utilizou-se o conjunto de programas EPI INFO versão $5.01 \mathrm{~b}^{7}$. Finalmente procedeu-se à análise multivariada pela regressão logística, utilizandose o programa MULTLR ${ }^{6}$. As RPC obtidas através desse procedimento serão referidas como RPC ajustadas.

\section{Resultados}

\section{Características da amostra}

Do total de 1.800 indivíduos para serem entrevistados, obteve-se informação de 1.459 , o que representa $80 \%$ do previsto. Na Tabela 1 encontra-se a distribuição dos indivíduos estudados segundo algumas variáveis sóciodemográficas. Houve predominância de indivíduos do sexo feminino (60\%), com menos de 40 anos $(61,5 \%)$ e casados $(60,2 \%)$. Dos entrevistados, $31,6 \%$ não
Tabela 1. Perfil sociodemográfico da amostra estudada $(n=1.459)$.

\begin{tabular}{|c|c|c|}
\hline Variável & $\mathbf{N}$ & $\%$ \\
\hline $\begin{array}{l}\text { Sexo } \\
\text { - masculino } \\
\text { - feminino }\end{array}$ & $\begin{array}{l}588 \\
871\end{array}$ & $\begin{array}{l}40,0 \\
60,0\end{array}$ \\
\hline $\begin{array}{l}\text { Idade } \\
\begin{aligned} & \cdot 14-19 \text { anos } \\
& \cdot 20-29 \text { anos } \\
& \cdot 30-39 \text { anos } \\
& \cdot 40-49 \text { anos } \\
& \cdot 50-59 \text { anos } \\
& \text { : } 60-69 \text { anos } \\
& \text {.70 e mais }\end{aligned}\end{array}$ & $\begin{array}{r}154 \\
332 \\
383 \\
242 \\
181 \\
111 \\
56\end{array}$ & $\begin{array}{r}10,6 \\
22,8 \\
26,3 \\
16,6 \\
12,4 \\
7,6 \\
3,8\end{array}$ \\
\hline $\begin{array}{l}\text { Estado civil } \\
\text { - solteiro } \\
\text { - casado } \\
\text { - separado } \\
\text { - viúvo }\end{array}$ & $\begin{array}{r}427 \\
879 \\
52 \\
101\end{array}$ & $\begin{array}{r}29,3 \\
60,2 \\
3,6 \\
6,9\end{array}$ \\
\hline $\begin{array}{l}\text { Religião } \\
\text { - católico } \\
\text { - espírita } \\
\text { - protestante } \\
\text { - outras } \\
\text { - ateu }\end{array}$ & $\begin{array}{r}1.082 \\
101 \\
94 \\
114 \\
68\end{array}$ & $\begin{array}{r}74,2 \\
6,9 \\
6,4 \\
7,8 \\
4,7\end{array}$ \\
\hline $\begin{array}{l}\text { Escolaridade } \\
\text { - analfabeto } \\
\text { - } 1^{2} \text { grau incompleto } \\
\text { - } 1^{2} \text { grau completo } \\
\text { - } 2^{\mathrm{Q}} \text { grau incompleto } \\
\text { - superior incompleto } \\
\text { - superior completo }\end{array}$ & $\begin{array}{r}70 \\
391 \\
245 \\
156 \\
307 \\
96 \\
193\end{array}$ & $\begin{array}{r}4,8 \\
26,8 \\
16,8 \\
10,7 \\
21,0 \\
6,6 \\
13,2\end{array}$ \\
\hline $\begin{array}{l}\text { Renda familiar } \\
\begin{aligned} \cdot 0-3 \quad \text { SM } \\
\cdot 4-6 \quad S M \\
\cdot 7-10 \quad S M \\
\cdot 11-20 \text { SM } \\
\cdot 21 \text { e + SM } \\
\text { - não informou }\end{aligned}\end{array}$ & $\begin{array}{r}507 \\
342 \\
205 \\
172 \\
84 \\
149\end{array}$ & $\begin{array}{r}34,7 \\
23,4 \\
14,1 \\
11,8 \\
5,8 \\
10,2\end{array}$ \\
\hline
\end{tabular}

$\mathrm{SM}=$ Salário mínimo.

completaram o primeiro grau e $34,7 \%$ referiram uma renda familiar igual ou menor que três salários mínimos. A grande maioria declarou-se católica $(74,2 \%)$.

\section{Consumo de álcool}

Declararam fazer uso de bebidas alcoólicas $52 \%$ dos entrevistados (Figura 1). A bebida mais consumida por esses indivíduos foi a cerveja, tendo sido referida por $88,8 \%$ daqueles que disseram fazer uso de álcool. A média de idade com que começaram a beber foi de 18,8 anos (desvio padrão $=4,96$ ), sendo que um quarto desses indivíduos o fez antes dos 16 anos e metade até os 18 anos. A idade mínima 
referida para o início de uso de álcool foi de 4 anos e a máxima de 50 anos.

A prevalência de consumidores na amostra cai, sobretudo, a partir dos 50 anos (Figura 2). A proporção de abstinentes foi maior entre as mulheres, os protestantes, os viúvos, aqueles de menor renda e menor escolaridade (Tabela 2). Observou-se ainda uma interação entre as variáveis renda e sexo, com uma associação mais forte da abstinência com niveis de renda inferiores entre os homens do que entre as mulheres.

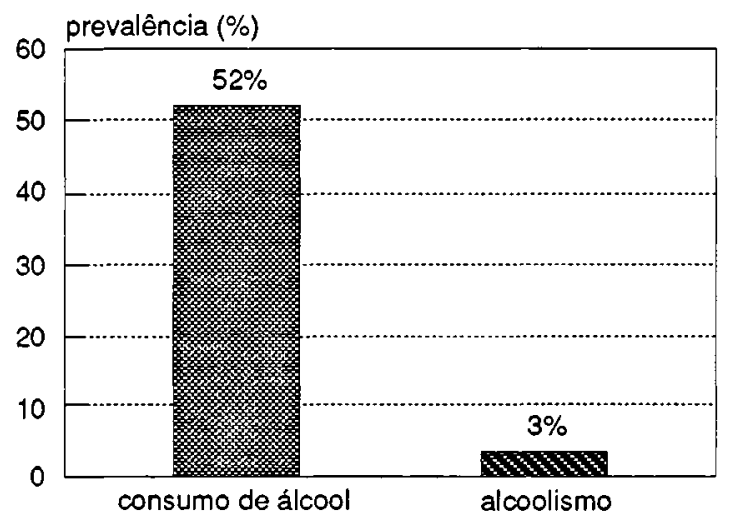

Figura 1. Prevalência de consumo de álcool e de alcoolismo na população estudada $(n=1.459)$.
Ao se estudar cada uma dessas variáveis separadamente, com as demais sob controle (RPC ajustada), apenas a escolaridade perdeu o nível de significância da associação com o consumo de bebida alcoólica, para um valor de p inferior a 0,05.

\section{Alcoolismo}

O estudo revelou uma prevalência de alcoolismo de $3 \%$, isto é, 44 pessoas responderam afirmativamente a 2 ou mais perguntas do teste CAGE. Entre o sexo masculino a prevalência foi de $4,9 \%$, enquanto entre o sexo feminino foi de 1,7\%. Esses achados, assim como as prevalências de CAGE positivo segundo variáveis sociodemográficas, encontram-se na Tabela 2.

Se considerarmos apenas aqueles entrevistados com 18 anos ou mais, a prevalência passa para $3,2 \%$. No caso dos maiores de 21 anos, a prevalência alcança $3,5 \%$.

Não se observou diferença entre a idade de início de consumo referida pelos indivíduos CAGE positivo e aqueles CAGE negativo $(18,51 \mathrm{e}$ 18,83 , Kruskal-Wallis $=0,937, p=0,33$ ).

Pelo estudo das RPC não ajustadas, o sexo masculino, a renda familiar superior a 3 salários mínimos e o grupo etário entre 30 e 49 anos mostraram-se positivamente associados com o alcoolismo (Tabela 3).

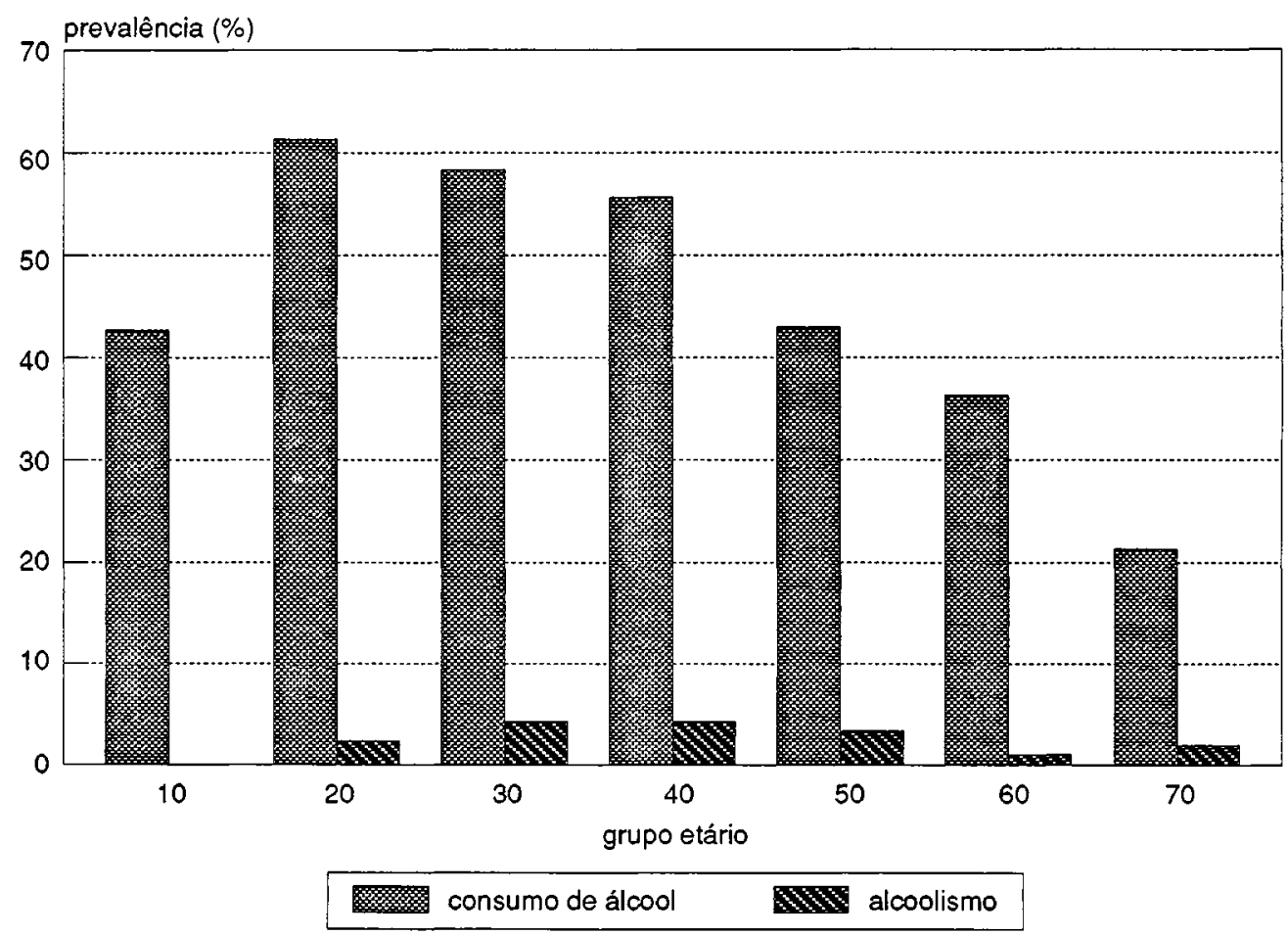

Figura 2. Prevalência de consumo de álcool e de alcoolismo segundo grupos etários, na população estudada $(n=1.459)$. 
Tabela 2. Consumo de álcool e alcoolismo segundo variáveis sóciodemográficas.

\begin{tabular}{|c|c|c|c|c|c|}
\hline \multirow[t]{2}{*}{ Variáveis } & \multicolumn{2}{|c|}{$\begin{array}{l}\text { Uso de } \\
\text { álcool }\end{array}$} & \multicolumn{2}{|c|}{ Alcoolismo } & \multirow{2}{*}{$\frac{\text { Tota }}{\mathrm{N}}$} \\
\hline & $N$ & $\%$ & $\mathbf{N}$ & $\%$ & \\
\hline \multicolumn{6}{|l|}{ Idade } \\
\hline $\begin{array}{l}.14-29 \text { anos } \\
.30-49 \text { anos } \\
.50 \mathrm{e}+\end{array}$ & $\begin{array}{l}269 \\
369 \\
131\end{array}$ & $\begin{array}{l}55,3 \\
57,4 \\
37,6\end{array}$ & $\begin{array}{r}9 \\
28 \\
7\end{array}$ & $\begin{array}{l}1,6 \\
4,5 \\
2,0\end{array}$ & $\begin{array}{l}486 \\
625 \\
348\end{array}$ \\
\hline \multicolumn{6}{|l|}{ Sexo } \\
\hline $\begin{array}{l}\text { - masculino } \\
\text { - feminino }\end{array}$ & $\begin{array}{l}376 \\
383\end{array}$ & $\begin{array}{l}63,9 \\
44,0\end{array}$ & $\begin{array}{l}29 \\
15\end{array}$ & $\begin{array}{l}4,9 \\
1,7\end{array}$ & $\begin{array}{l}588 \\
871\end{array}$ \\
\hline \multicolumn{6}{|l|}{ Estado civil } \\
\hline $\begin{array}{l}\text { - solteiro } \\
\text { - casado } \\
\text { - separado } \\
\text { - viúvo }\end{array}$ & $\begin{array}{r}238 \\
463 \\
29 \\
29\end{array}$ & $\begin{array}{l}55,7 \\
52,7 \\
55,8 \\
28,7\end{array}$ & $\begin{array}{r}10 \\
31 \\
0 \\
3\end{array}$ & $\begin{array}{l}2,3 \\
3,5 \\
0 \\
3,0\end{array}$ & $\begin{array}{r}427 \\
879 \\
52 \\
68\end{array}$ \\
\hline \multicolumn{6}{|l|}{ Religião } \\
\hline - católico & 574 & 53,0 & 35 & 3,2 & 1.082 \\
\hline - espírita & 58 & 57,4 & 3 & 3,0 & 101 \\
\hline - protestante & 34 & 36,2 & 3 & 3,2 & 94 \\
\hline - outras & 48 & 42,1 & 2 & 1,8 & 114 \\
\hline - ateu & 45 & 66,2 & 1 & 1,5 & 68 \\
\hline \multicolumn{6}{|l|}{ Escolaridade } \\
\hline - analfabeto & 28 & 40,0 & 1 & 1,4 & 70 \\
\hline - $1^{2}$ grau incompleto & 178 & 45,5 & 9 & 2,3 & 391 \\
\hline - $1^{8}$ grau completo & 108 & 44,1 & 6 & 2,4 & 245 \\
\hline - $2^{9}$ grau incompleto & 77 & 49,4 & 3 & 1,9 & 156 \\
\hline - $2^{8}$ grau completo & 176 & 57,3 & 13 & 4,2 & 307 \\
\hline - superior incompleto & $\begin{array}{r}66 \\
125\end{array}$ & 68,7 & $\begin{array}{l}5 \\
7\end{array}$ & 5,2 & 96 \\
\hline • superior completo & & & & & 190 \\
\hline \multicolumn{6}{|l|}{ Renda familiar } \\
\hline - $0-3 \quad S M$ & 224 & 44,2 & 10 & 2,0 & 507 \\
\hline - 4-6 SM & 188 & 55,0 & 12 & 3,5 & 342 \\
\hline - 7-10 SM & 120 & 58,5 & 9 & 4,4 & 205 \\
\hline$\cdot 11 e+S M$ & 164 & 64,1 & 10 & 3,9 & 256 \\
\hline
\end{tabular}

$\mathrm{SM}=$ Salário mínimo.

Nas RPC ajustadas por regressão logística, a associação com a renda perdeu a significância estatística.

\section{Teste CAGE}

Dos 759 usuários de bebidas alcoólicas, 70 $(9,2 \%)$ afirmaram sentir necessidade de reduzir a ingestão de bebidas alcoólicas (questão $\mathrm{n}^{\circ} 1$ ). Entretanto, entre aqueles com teste CAGE positivo (duas ou mais respostas afirmativas), essa proporção foi de $84,1 \%$ contra $4,6 \%$ daqueles identificados como não alcoolistas segundo os critérios diagnósticos da pesquisa.

Com relação ao fato de terceiros criticarem o modo pelo qual esses 759 indivíduos usavam o álcool (questão $\left.n^{2} 2\right), 46(6,1 \%)$ referiram sofrer
Tabela 3. Associação de variáveis sóciodemográficas com o uso de bebidas alcoólicas e alcoolismo através das razōes de produtos cruzados (RPC) simples e ajustadas por modelo logístico.

\begin{tabular}{|c|c|c|c|c|}
\hline \multirow[t]{2}{*}{ Variável } & \multicolumn{2}{|c|}{ Uso de álcool } & \multicolumn{2}{|c|}{ Alcoolismo } \\
\hline & $\begin{array}{c}\mathrm{RPC} \\
\text { simples }\end{array}$ & $\begin{array}{c}\mathrm{RPC} \\
\text { ajustada }\end{array}$ & $\begin{array}{c}\text { RPC } \\
\text { simples }\end{array}$ & $\begin{array}{c}\text { RPC } \\
\text { ajustada }\end{array}$ \\
\hline \multicolumn{5}{|l|}{ Idade } \\
\hline $\begin{array}{l}\text {. 14-29 anos } \\
\text {-30-49 anos } \\
.50 \mathrm{e}+\end{array}$ & $\begin{array}{l}1 \\
1,09 \\
0,49^{* * *}\end{array}$ & $\begin{array}{l}1 \\
1,11 \\
0,55^{\star * *}\end{array}$ & $\begin{array}{l}1 \\
2,5^{\star \star} \\
1,09\end{array}$ & $\begin{array}{l}1 \\
2,29^{*} \\
1,10\end{array}$ \\
\hline \multicolumn{5}{|l|}{ Sexo } \\
\hline $\begin{array}{l}\text { - masculino } \\
\text { - feminino }\end{array}$ & $\begin{array}{l}2,27^{* * \star} \\
1\end{array}$ & $\begin{array}{l}1,66^{* \pm *} \\
1\end{array}$ & $\begin{array}{l}2,94^{* * *} \\
1\end{array}$ & $\begin{array}{l}2,76^{* * *} \\
1\end{array}$ \\
\hline \multicolumn{5}{|l|}{ Estado civil } \\
\hline $\begin{array}{l}\text { - viúvo } \\
\text { - outras }\end{array}$ & $\begin{array}{l}0,35^{* * *} \\
1\end{array}$ & $\begin{array}{l}0,59^{* *} \\
1\end{array}$ & $\begin{array}{l}0,98 \\
1\end{array}$ & $t_{1}^{1,10}$ \\
\hline \multicolumn{5}{|l|}{ Religião } \\
\hline $\begin{array}{l}\text { - protestante } \\
\text { - outras }\end{array}$ & $\begin{array}{l}0,50^{* * *} \\
1\end{array}$ & $\begin{array}{l}0,52^{\star \star *} \\
1\end{array}$ & $\begin{array}{l}1,05 \\
1\end{array}$ & $\begin{array}{l}1,27 \\
1\end{array}$ \\
\hline \multicolumn{5}{|l|}{ Escolaridade } \\
\hline $\begin{array}{l}\text { incompleto } \\
\text { - } 1^{2} \mathrm{grau} \\
\text { completo e }\end{array}$ & $0,65^{\star \star \star}$ & 0,97 & 0,63 & 0,84 \\
\hline mais & 1 & 1 & 1 & 1 \\
\hline \multicolumn{5}{|l|}{ Renda familiar } \\
\hline $\begin{array}{l}\cdot \text { até } 3 \text { SM } \\
.4 e+S M\end{array}$ & $\begin{array}{l}0,56^{* * *} \\
1\end{array}$ & $\begin{array}{l}0,25^{* * *} \\
1\end{array}$ & $\begin{array}{l}0,50^{*} \\
1\end{array}$ & $\begin{array}{l}0,57 \\
1\end{array}$ \\
\hline
\end{tabular}

Obs: os valores 1 nas colunas das RPC indicam que a categoria foi tomada como referência.

* $0,08>p>0,05$

$* 0,05>p>0,01$

*** $p<0,01$

essas críticas, sendo que entre os CAGE positivos isso ocorreu em $65,9 \%$ contra $2,4 \%$ dos CAGE negativos.

Afirmaram sentirem-se culpados pelo modo como bebiam (questão $n^{2} 3$ ), 5,5\% dos usuários de bebidas alcoólicas. No entanto, esse sentimento ocorria em $79,5 \%$ dos considerados alcoolistas contra apenas $1 \%$ dos não alcoolistas.

Finalmente, encontrou-se que $3,6 \%$ dos entrevistados bebiam pela manhã, assim que acordavam (questão $n^{2} 4$ ), sendo que essa prática foi verificada em $31,8 \%$ dos indivíduos CAGE positivos e 1,8\% dos CAGE negativos. Esse comportamento é característico dos indivíduos que já apresentam distürbio de dependência de álcool ${ }^{2}$ e decorre da necessidade de manter o nível de alcoolemia, evitando assim os sintomas de abstinência. Encontrou-se 13 entrevistados ( 8 homens e 5 mulheres) que referiram esse comportamento, mas responderam negativamente às outras 3 perguntas do teste. 


\section{Discussão}

Se forem levados em conta os poucos estudos nacionais que estimaram prevalência de alcoolismo em população geral, os presentes achados situam-se no extremo inferior do espectro de resultados. Se forem considerados como alcoolistas também os indivíduos que, apesar de não alcançarem 2 pontos no teste CAGE, responderam afirmativamente à questão referente ao consumo de álcool pela manhã, a prevalência passaria de $3 \%$ para $3,9 \%$, sendo de $6,3 \%$ em homens e de $2,3 \%$ em mulheres.

Embora seja recomendável a correção de prevalências obtidas por instrumentos de "screening" a partir dos erros de classificação observados nos procedimentos de validação, optou-se por não adotar este procedimento. A decisão deve-se ao fato do valor preditivo descrito na literatura não se aplicar ao presente caso (estudo em população geral) já que essa medida varia com a prevalência do fenômeno estudado. Mesmo que fossem utilizadas fórmulas de correção que usam como parâmetros a prevalência obtida pelo teste e as proporções de falso-positivo e falso-negativo (que não variam com a prevalência), teríamos que aceitar, na presente investigação, valores de sensibilidade e de especificidade obtidos em estudos realizados em condições distintas. Para reforçar esse ponto de vista, esclarece-se que essa tentativa de correção com dados de outros estudos foi por nós implementada em caráter experimental, resultando em prevalências negativas de alcoolismo.

No Brasil a prevalência encontrada por Santa$\mathrm{na}^{24}$ é a que mais se aproxima dos presentes achados. Entretanto, comparaçōes entre os estudos conduzidos no pais esbarram em questões metodológicas, como a utilização de instrumentos diagnósticos distintos. Além desse aspecto, a utilização do teste CAGE como critério de alcoolismo merece cautela por se tratar de um questionário de "screening" e não de diagnóstico final, necessitando também de estudos de validação em âmbito populacional.

Também chamou atenção o fato da proporção de indivíduos que consumiam álcool (52\%) ser bastante inferior ao relatado em diversos países: $90 \%$ nos EUA $^{25}, 87 \%$ na Austrália ${ }^{19}, 83 \%$ no Canadá 26 e $75 \%$ no Equador ${ }^{1}$. No entanto, houve semelhança com a Colômbia e México: $51 \%^{1,16}$. A maior proporção de mulheres entre os entrevistados $(60 \%)$ não parece ser suficiente para explicar essas discrepâncias.

Os resultados do inquérito estão de acordo com a literatura no que se refere à predominância do alcoolismo crônico no sexo masculino e na faixa dos 30 a 49 anos de idade. Trata-se de um achado que vem sendo consistentemente descrito, mas cuja causa ainda não está devidamente esclarecida. Hipóteses têm sido construídas sobre pressupostos biológicos, sociais, econômicos, culturais. Fala-se, entre outras coisas, no papel social da mulher, na identificação da virilidade com a capacidade de beber, na utilização do álcool como ansiolítico entre os homens, na moral restritiva e estigmatizante do consumo de álcool em mulheres ${ }^{22}$.

O padrão de consumo segundo o sexo vem sendo objeto de discussão pela epidemiologia do alcoolismo. Tem-se descrito aumento na proporção de mulheres que consomem bebidas alcoólicas no período pós-guerra ${ }^{20}$. Além disso, prevalências mais elevadas no sexo feminino de uso diário de álcool, de embriaguez semanal, de "bebedores leves" vêm sendo observadas com alguma consistência ${ }^{22}$. Entretanto, esse aspecto não foi explorado na presente investigação.

Se os resultados observados quanto às variáveis sexo e grupo etário foram coerentes com os dados da literatura, o mesmo não pode ser dito com relação às chamadas variáveis socioeconômicas $\mathrm{e}$ culturais. No Brasil ${ }^{22}$, 23 , prevalências mais elevadas de alcoolismo foram descritas para as menores faixas de renda per capita e de escolaridade. Entretanto, o presente inquérito mostrou associação entre o uso de álcool e os níveis de renda e escolaridade mais elevados.

Polich e Kaebler ${ }^{20}$ ao sumarizarem os resultados de inquéritos realizados nos EUA, entre 1964 e 1979, assinalam que as variáveis socioeconômicas se associam com o uso de álcool e com os problemas a ele relacionados. Segundo esses resultados, a abstinência é mais freqüente em indivíduos de padrão social e econômico mais baixos. Entretanto, entre aqueles que consomem bebidas alcoólicas, os problemas relacionados com - álcool são mais freqüentes nos niveis socioeconômicos inferiores.

Os presentes resultados estão, em parte, de acordo com aqueles sumarizados por Polich e Kaebler ${ }^{20}$ pois houve associação entre a abstinência e os níveis de renda inferiores. Entretanto, não é possível afirmar o mesmo quanto ao alcoolismo. Apesar das RPC ajustadas sempre apontarem para uma relação entre alcoolismo e níveis de renda e de escolaridade mais baixos, não foi possível rejeitar a hipótese nula de ausência de associação. Apenas a variável renda mostrou uma significância estatística entre 0,05 e 0,08 referente a RPC não ajustada, isto é, não controlada para variáveis potencialmente "confounding".

Para melhor responder à pergunta sobre associação entre nível socioeconômico e alcoolismo no Brasil são necessários estudos especialmente planejados e desenhados para este objetivo, e não a uti- 
lização de dados aproveitados de inquéritos, como tem sido feito até então. Dados assim gerados ressentem-se, entre outras coisas, de um número suficiente de alcoolistas para constituir grupos adequados de comparação. Com isso, análises que utilizam estratificação e modelos multivariados para o controle de variáveis "confounding" ficam prejudicadas e os testes estatísticos perdem poder, isto é, rejeitam mais facilmente hipóteses verdadeiras.

Por outro lado, resultados de investigações conduzidas em outros países devem ser consideradas com cautela. As peculiaridades da estratificação social e econômica no Brasil não devem ser menosprezadas, e variáveis como escolaridade, renda, índices de posse de bens materiais nem sempre guardam relações homogêneas como as observadas em outras populaçz̃es, sobretudo do primeiro mundo.

ALMEIDA, L. M. de \& COUTINHO, E. da S. F. [Prevalence of the consumption of alcoholic beverages and of alcoholism in an urban region of Brazil. Rev. Saúde Públlica, 27: 23-9, 1993. An epidemiological survey was carried out for the purpose of estimating the use of psychoactive substances and the prevalence of alcoholism. The target population consisted of people over 13 years old living in a district of Rio de Janeiro city - Brazil. A random sample of 1,459 people was researched. Data on the use of alcohol and on alcoholism are presented. The diagnosis of this latter, based upon the CAGE Test, showed that $51 \%$ used alcohol and $3 \%$ were suspected of alcoholism: $4.9 \%$ and $1.7 \%$ among men and women, respectively. The greatest prevalence of the use of alcohol and of alcoholism was found among men between 30 and 49 years of age. Abstinence from alcohol was more frequent among widowed, Evangelical and low-income groups.

Keywords: Alcoholism, epidemiology. Alcohol drinking, epidemiology.

\section{Referências Bibliográficas}

1. AGUILAR, Z. E. Prevalencia del uso indebido de alcohol, tabaco y drogas en la población ecuatoriana. In: Organización Panamericana de la Salud. Abuso de drogas. Wash ington, D. C., 1990. p. 48-52 (OPAS - Publ. cient., 522).

2. AMERICAN PSYCHIATRIC ASSOCIATION. Manual de diagnóstico e estatística de distúrbios mentais, DSM III-R. São Paulo, Editora Manole Ltda, 1989.

3. AZOUBEL NETO, D. Estado actual de la epidemiologia del alcoholismo y problemas del al cohol en Brasil. In: Horwitz, J. et al. Bases para una epidemiologia del alcoholismo en America Latina. Buenos Aires, ACTA, 1967.p. 61-126.

4. BARESFORD, T. et al. A Comparison of CAGE questionnaire and computer-assisted laboratory profiles in screening for covert alcoholism. Lancet, 336: 482-5, 1990.

5. CABERNITE, L. O alcoolismo no Brasil e as dificuldades na área-epidemiologia e prevenção. J. Bras. Psiq., 31: 89-112, 1982.

6. CAMPOS FILHO, N. \& FRANCO, E. L. A microcomputer program for multiple logistic regression by uncondition. al and conditional maximum likelihood methods. Am.J. Epidemiol., 129: 439-44, 1989.

7. DEAN, A. G. et al. Epi Info, version 5.016 Georgia, USD, Incorporated, 1990.

8. GALVIS, Y. T. \& MURRELLE, L. Consumo de substancias que producen dependencia en Colombia. In: Organización Panamericana de la Salud. Abuso de drogas. Washington, D. C., 1990. p. 17-28. (OPAS-Publ. cient. 522).

9. INSTITUTO NACIONAL SOBRE ALCOHOLISMO. Departamento de Investigación (Costa Rica). El alcoholis. mo en la ciudad de Alajuela. San José, 1983.

10. INSTITUTO NACIONAL SOBRE ALCOHOLISMO. Departamento de Investigación (Costa Rica). El alcoholismo en la area programática del Canton de Parrita. San José, 1983.

11. INSTITUTO NACIONAL SOBRE ALCOHOLISMO. Departamento de Investigación (Costa Rica). El alcoholismo en la ciudad de Quespos. San José, 1983.

12. KING, M. At risk drinking among general practice attenders: validation of the CAGE questionnaire. Psychol. Med. 16: 213-7, 1986.

13. LUZ JR., E. Estudo da prevalência do alcoolismo numa Vila Marginal de Porto Alegre. Rev. Med. ATM., 9: 407-32, 1984.

14. MANFIELD, D. et al. The CAGE questionnaire: validation of a new alcoholism screening instrument. Am. J. Psychiat., 131: 1121-3, 1974.

15. MASUR, J. \& MONTEIRO, M. Validation of the CAGE alcoholism screening test in Brazilian Psychiatry Inpatient Hospital Setting. J. Biol. Res., 16: 215-8, 1983.

16. MEDINA -MORA, M. E. et al. Situación epidemiologica del abuso de drogas en Mexico. In: Organización Panamericana de la Salud. Abuso de drogas. Washington, D. C. 1990. p. 3-16 (OPAS - Publ. cient., 522).

17. MINISTÉRIO DA SAUDE. Programa Nacional de Controle dos Problemas Relacionados com o Consumo de Álcool (PRONAL). Brasília, 1988. [Mimiografado].

18. MURILLO, E. A. Abuso de drogas en Costa Rica: recopilacion de varios estudos. In: Organización Paramericana de la Salud. Abuso de drogas. Washington, D. C., 1990. p. 41-7 (OPAS - Publ. cient. 522).

19. NATIONAL HEART FOUNDATION OF AUSTRALIA. Australian Institute of Health. Risk Factor Prevalence Study, Survey n. 3, 1989.

20. POLICH, M. J. \& KAEBLER, C. T. Sample surveys and the epidemiology of alcoholism. In: Shuckit, M. A. Alcohol patterns and problems. New Brunswick, Rutgers University Press, 1985. p. 43-78.

21. RUEFF, B. et al. Dèpistage de Malades "alcooliques" per l'autoquestionnaire Systematic DETA. Parmides Consultants Hospitaliers. Presse Méd., 18: 1654-65, 1989.

22. SANTANA, V. S. \& ALMEIDA FILHO, N. Aspectos epidemiológicos do alcoolismo. In: Ramos, S.P. Alcoolismo hoje. Porto Alegre, Ed. Artes Médicas, 1987. p. 29-44.

23. SANTANA, V. S. et al. Prevalência de alcoolismo em uma área urbana de Salvador-Bahia II-variáveis sócioeconômicas. J. Bras. Psiq., 38: 75-81, 1989.

24. SANTANA, V. S. Estudo epidemiológico das doenças mentais em um Bairro de Salvador. Salvador, Instituto de Saúde do Estado da Bahia, 1982.

25. SCHUKIT, M. A. Abuso de álcool e drogas. Porto Alegre, Ed. Artes Médicas, 1991.

26. SMART, R. Abuso de drogas en Ontario, Canadá. In: Organjzación Panamericana de la Salud. Abuso de drogas. Washington, D. C., 1990. p. 29-40 (OPAS - Publ. cient. 522).

Recebido para publicaçäo em 16.7.1992 Reapresentado em 4.12.1992 Aprovado para publicaçāo em 21.12.1992 